\title{
Comparison of a novel handheld accelerometer-based navigation system and conventional instrument for performing distal femoral resection in total knee arthroplasty: a randomized controlled trial
}

\author{
Xingquan Xu ${ }^{1,2 \#}$, Peilai Liu ${ }^{3 \#}$, Zhenfeng Yuan ${ }^{4 \#}$, Dawei Wang ${ }^{4}$, Qunshan Lu $^{3}$, Zhe Zhang ${ }^{1,2}$, Qing Jiang ${ }^{1,2}$, \\ Dongquan $\mathrm{Shi}^{1,2}$
}

${ }^{1}$ State Key Laboratory of Pharmaceutical Biotechnology, Department of Sports Medicine and Adult Reconstructive Surgery, Nanjing Drum Tower Hospital, The Affiliated Hospital of Nanjing University Medical School, Nanjing 210008, China; ${ }^{2}$ Joint Research Center for Bone and Joint Disease, Model Animal Research Center (MARC), Nanjing University, Nanjing 210093, China; ${ }^{3}$ Qilu Hospital of Shandong University, Jinan 250012, China; ${ }^{4}$ Liaocheng People's Hospital, Liaocheng 252000, China

Contributions: (I) Conception and design: D Shi, Q Jiang; (II) Administrative support: D Shi, Q Jiang; (III) Provision of study materials or patients: D Wang, Q Lu, Z Zhang; (IV) Collection and assembly of data: D Wang, Q Lu, Z Zhang, X Xu; (V) Data analysis and interpretation: X Xu; (VI) Manuscript writing: All authors; (VII) Final approval of manuscript: All authors.

\#These authors contributed equally to this work.

Correspondence to: Dr. Qing Jiang; Dongquan Shi. State Key Laboratory of Pharmaceutical Biotechnology, Department of Sports Medicine and Adult Reconstructive Surgery, Drum Tower Hospital, School of Medicine, Nanjing University, 321 Zhongshan Road, Nanjing 210008, China.

Email: qingj@nju.edu.cn; shidongquan1215@163.com.

Background: This prospective study aimed to compare the efficacy of a novel, hand-held, accelerometerbased navigation system (i-JOIN knee navigation system) for distal femoral resection in total knee arthroplasty (TKA) with conventional instrument.

Methods: A multi-center, double-blinded, randomized controlled trial (RCT) was conducted. A total of 79 consecutive patients scheduled for primary TKA were enrolled and divided into navigation group (39 patients) and conventional group (40 patients). Post-operative mechanical and component position were evaluated through full-leg weight bearing X-ray. Pre-operatively and 1 week post-operatively, adverse events were recorded. Intraoperative surgical time and blood loss were also recorded.

Results: The mean outlier of $180^{\circ}$ neutral mechanical axis was $1.60^{\circ}\left(\mathrm{SD} 1.11^{\circ}\right)$ in navigation group and $2.30^{\circ}\left(\mathrm{SD} 2.06^{\circ}\right)$ in conventional group $(\mathrm{P}=0.0917)$. Thirty-eight patients $(97.4 \%)$ in navigation group and 35 patients $(87.5 \%)$ in conventional group had an alignment which was $\leq 3^{\circ}$ away from the neutral mechanical axis $(\mathrm{P}=0.2007)$. $\alpha$ angle between the navigation group and conventional group was not statistically different $\left(89.81^{\circ}\right.$ vs. $\left.89.76^{\circ}, \mathrm{P}>0.05\right)$, as well as adverse events rate post-operatively. The operative time of navigation group was significantly longer than that of control group $(114.54 \pm 35.34$ vs. $100.33 \pm 28.38 \mathrm{~min}, \mathrm{P}=0.0493)$, whereas the intraoperative blood loss was not significantly different.

Conclusions: i-JOIN knee navigation system had equivalent results for distal femoral resection in TKA compared with the conventional technique.

Keywords: Total knee arthroplasty (TKA); i-JOIN knee navigation system; conventional instrument; mechanical axis, accuracy

Submitted Jul 09, 2019. Accepted for publication Sep 26, 2019.

doi: $10.21037 /$ atm.2019.10.55

View this article at: http://dx.doi.org/10.21037/atm.2019.10.55 


\section{Introduction}

Lower limb alignment and components position play a crucial role in successful total knee arthroplasty (TKA). Malalignment of the lower limb, particularly in the coronal plane (varus/valgus) could result in the risk of abnormal wear and implant loosing (1-3). Indeed, several studies have reported early TKA failure because of unsatisfactory implant alignment $(4,5)$. Until now on, most of the surgeons still aim to gain neutral lower limb alignment in TKA. And, variance not within $\pm 3^{\circ}$ is defined as "inadequate alignment".

Distal femoral resection influences the lower limb mechanical axis significantly. Conventional intramedullary guide is the most commonly used method to guide the position of distal femoral cutting block. However, this method may be inaccurate $(6,7)$, especially when used for patients with femoral extra-articular deformity (8). Moreover, it is believed violation of the intramedullary femoral canal and subsequent use of intramedullary instruments is one of the most invasive parts of TKA. Several methods have been developed as alternatives to intramedullary instrument. Among those, portal accelerometer-based navigation system was meant to improve lower limb mechanical axis in TKA with the features of convenient and accurate. This kind of device has lots of advantages including portability without a large of computer, simplicity, and accuracy $(9,10)$. This kind of system also avoids violation of the intramedullary femoral canal and may reduce the possibility of blood loss and fat embolism. Several studies have showed encouraging results by using portable accelerometer-based navigation system in TKA. An accelerometer-based navigation system, iAssist, was reported with improved postoperative mechanical axis without significantly increasing surgical time (11). Compared with conventional computer assisted navigation system (CAS), iAssist had the same accurate and shorter duration of surgery (12). Another accelerometer-based portal navigation system, KneeAlign, also showed better accuracy towards conventional instrument in some studies $(13,14)$.

A novel handheld accelerometer-based navigation system, i-JOIN knee navigation system [i-JOIN (Shanghai) medical technology co., LTD], has been developed with the characteristics of simplicity and ease of use. We hypothesized that i-JOIN knee system had lots of advantages over conventional instrument in distal femoral resection during TKA. The purpose of this study was to evaluate the accuracy of the femoral component alignment by using i-JOIN knee navigation system in TKA and to investigate whether it can reduce blood loss and surgical time compared to a conventional instrument.

\section{Methods}

\section{Patient selection}

Between Jul 2017 and Dec 2018, a prospective multicenter, double-blinded, randomized controlled trial (RCT) was conducted. The study was approved by the authors' Institutional Review Boards (No.2017-119-02), and informed consent was signed by all the participants. The study has been approved on Clinical Trial Registry (ID: ChiCTR1900023608). Patients with primary knee osteoarthritis, aged from 18 to 80 were recruited. Exclusion criteria included patients who were unwilling to participate in this study, women who are pregnant or breastfeeding, and patients with previous distal femoral osteotomy or limited hip motion or serious knee ligament laxity (lower extremity axis deviation $>15^{\circ}$ ). Total 80 patients scheduled for primary TKA were enrolled in three hospitals (Nanjing Drum Tower Hospital, Qilu Hospital of Shandong University, and Liaocheng People's Hospital) and 1:1 randomized into two groups, the navigation group and the conventional group. One patient in navigation group was excluded because of insufficient instruments during the operation. The remaining 79 patients comprised the study population. Computer-generated random number table was used for randomization and allocation. Patients and the assessor responsible for evaluating the functional outcomes in this trial were kept blinded, while the surgeons were informed of the randomization results on the day of surgery by opening a sealed envelope.

\section{Surgical procedures}

General anesthesia was performed in both groups. Prophylactic antibiotics were administered 30 minutes before the operation and discontinued within 24 hours after surgery. All the operations were performed by using a posterior-stabilized TKA prosthesis (Genesis II, Smith \& Nephew, USA). The target overall mechanical axis of the limb was $180^{\circ}$. The desired coronal femoral component alignment was $90^{\circ}$. A conventional extramedullary guide was used for tibial resection in both groups. Proximal tibia resection was perpendicular to the mechanical axis and the desired posterior tibial slope was $3^{\circ}$. i-JOIN knee 

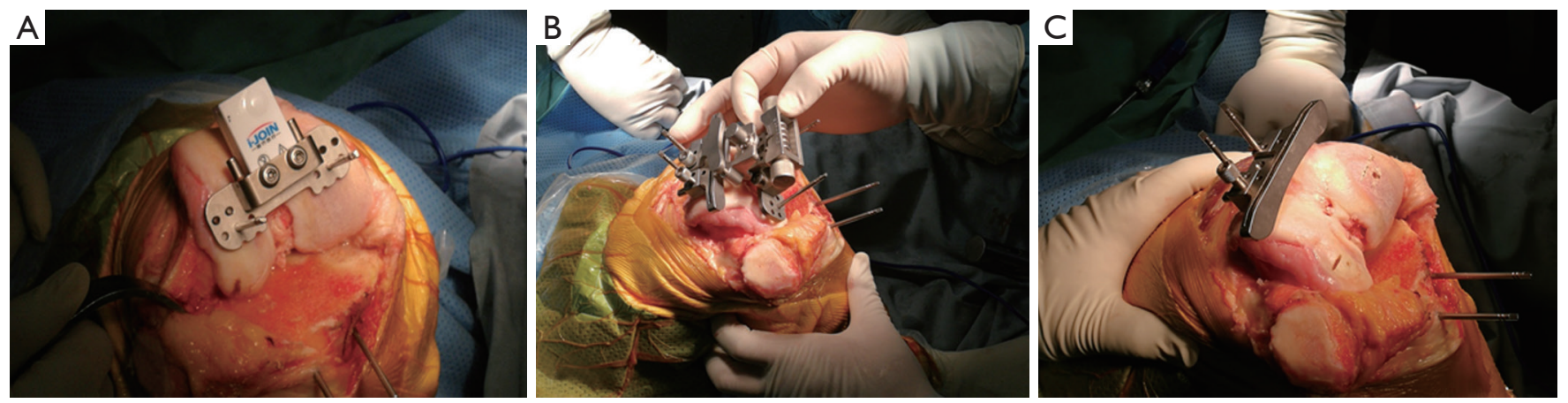

Figure 1 The process i-JOIN knee navigation system guided the position of the distal femoral cutting block. (A) Stabilize the reference sensor to the distal femur. (B) Adjust the cutting block using the real-time data. (C) The final position of the cutting block.

navigation system was used for the distal femoral resection in 39 patients (navigation group), and a conventional intramedullary guide was used in 40 patients with patientspecific femoral valgus angle that was measured on full length anteroposterior hip to ankle weight-bearing radiographs preoperatively (conventional group). i-JOIN knee navigation system is a portal, accelerometer-based navigation device which consists of a display console (an i-Pad) and reference sensor. The display console and reference sensor are connected by blue-tooth. During the procedure, the reference sensor was stabilized to the distal femur to navigate the femoral head during movement of the hip and provided real-time feedback to the display console (Figure 1A). A device was used to guide the cutting block position according to the real-time data calculated and displayed by the console (Figure 1B,C). The remaining femoral resection procedures were identical in the two groups. Physiotherapy was started on the second day postoperatively.

\section{Measurements}

Intraoperative data collection included operative time and blood loss. Full length anteroposterior hip to ankle weight-bearing radiographs were obtained three days postoperatively. The outlier of the whole limb mechanical axis was determined by calculating the angle deviating from the $180^{\circ}$ Hip-Knee-Ankle (HKA) angle. The rate that outlier of mechanical axis $< \pm 3^{\circ}$ was also analyzed (Figure $2 A$ ). The coronal alignment of the femoral component was determined by evaluating the medial angle ( $\alpha$ angle) formed by the mechanical axis of the femur and the tangent of the distal femoral condyle (Figure 2B). Adverse events including anemia, deep venous thrombosis (DVT), pulmonary embolism (PE), infection, and the abnormal liver function test were also noted one week post-operatively. And, the total adverse events rates were calculated.

\section{Statistical analysis}

The primary outcome of the present study was the rate that outlier of mechanical axis $< \pm 3^{\circ}$. The following formula was used to calculate sample size in each group.

$$
n=\frac{\left(\mathrm{Z}_{\alpha}+\mathrm{Z}_{\beta}\right)^{2}\left[\mathrm{P}_{\mathrm{C}}\left(1-\mathrm{P}_{\mathrm{C}}\right)+\mathrm{P}_{\mathrm{T}}\left(1-\mathrm{P}_{\mathrm{T}}\right)\right]}{\left[\Delta-\left(\mathrm{P}_{\mathrm{T}}-\mathrm{PC}\right)\right]^{2}}
$$

Parameters were setted as follows: $\mathrm{P}=97.5 \%, \delta=0.1,1-\beta$ $=0.80$, and $\alpha=0.05$. A power analysis performed for primary outcome suggested that a sample size of 39 patients in each cohort was required to provide appropriate power $(1-\beta=0.80)$, allowing a type I error of 0.05 for a two-sided test. Forty subjects were enrolled in each group in case samples dropping out during the study. Cochran-MantalHaenszel (CMH) test were used to calibrate the bias caused by multi-centers. To analyze differences between the two groups, Student's t-test and Mann-Whitney U test were respectively adopted for continuous variables with normal and non-normal distribution. As to categorical variables, the chi-square test or Fisher's exact test were used. Statistical significance was set at $\mathrm{P}<0.05$. Statistical analysis was conducted with the use of Statistical Analysis System (SAS) Version 9.4 software offered by SAS Institute Incorporated.

\section{Manuscript preparation}

The work has been reported in line with Consolidated Standards of Reporting Trials (CONSORT) Guidelines. 


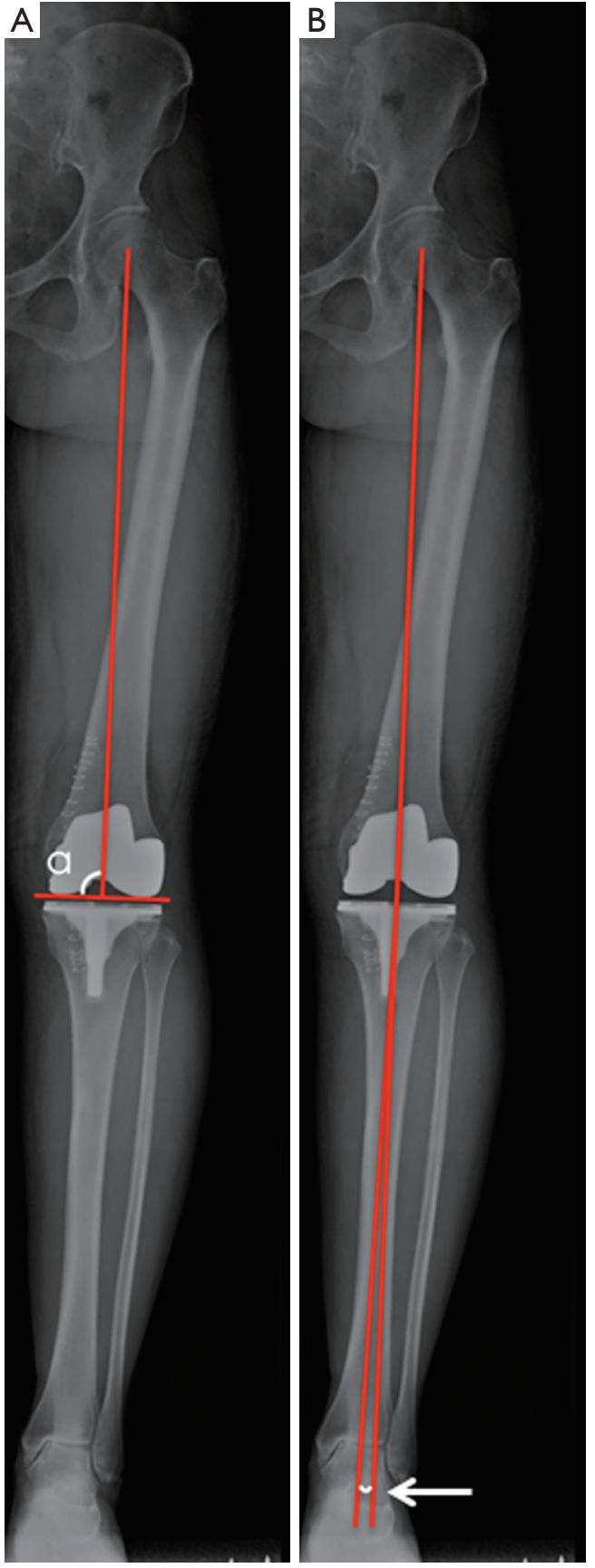

Figure 2 Radiographic measurements of the mechanical axis post operatively. (A) $\alpha$ angle of the operated knee. (B) angle deviating from the $180^{\circ}$ targeted HKA angle (arrow).

\section{Results}

No significant differences were detected between the two groups in their demographics, including age, gender, affected side, allergic history and contaminant diseases
(P>0.05, Table 1).

The mean outlier of the whole limb mechanical axis in navigation group was $1.60^{\circ}\left(\mathrm{SD} 1.11^{\circ} ; 0.07^{\circ}\right.$ to $\left.5.06^{\circ}\right)$, with 38 patients $(97.4 \%)$ having a variance within $\pm 3^{\circ}$. While, the mean outlier of the mechanical axis in control group was $2.30^{\circ}$ (SD $2.06^{\circ} ; 0.09^{\circ}$ to $11.01^{\circ}$ ), with 35 patients $(87.5 \%)$ having a variance within $\pm 3^{\circ}$. However, the differences were not statistically significant $(\mathrm{P}>0.05$, Table 2). $\alpha$ angle between navigation group and control group was not statistically significant $\left(89.81^{\circ}\right.$ vs. $89.76^{\circ}, \mathrm{P}=0.9922$, Table 2$)$.

The operative time of navigation group was significantly longer than that of control group $(114.54 \pm 35.34$ vs. $100.33 \pm 28.38 \mathrm{~min}, \mathrm{P}=0.0493$, Table 3). The intraoperative blood loss was not significantly different between the two groups $(105.13 \pm 58.66$ vs. $113.00 \pm 71.55 \mathrm{~mL}, \mathrm{P}=0.8825$, Table 3). The rate of patients developing adverse events was not statistically different between navigation (59\%) and control groups $(67.5 \%)(\mathrm{P}=0.4319$, Table 3$)$. The anemia rate in navigation group $(30.8 \%)$ was significantly lower than that in control group (55.0\%) $(\mathrm{P}=0.0297$, Table 3). One patient in control group was detected with $\mathrm{PE}$ (Table 3).

\section{Discussion}

The most significant finding of the study is that the mechanical axis of the operated knee was comparable between patients who had undergone TKA with i-JOIN knee navigation system and conventional intramedullary device.

Unlike the current results, several studies using similar navigation devices have showed much more accurate restoration of mechanical axis compared to conventional instruments $(11,13-18)$. However, most of those studies did not mention the femoral valgus angle when using conventional intramedullary rod for distal femoral resection $(11,13,14,18)$. Some studies just set the femoral valgus angle at $6^{\circ}(16,17)$. While, the current research used patient specific valgus angle aimed to maximally make sure that the distal femoral resection perpendicular to the mechanical axis and to reduce the bias caused by multicenters. Another concern was the targeted lower limb alignment by using i-JOIN. In the present study, the target overall mechanical axis of the limb was $180^{\circ}$ both in i-JOIN and conventional group. We chose mechanical alignment in performing TKA procedure to restore a neutral mechanical axis. Nowadays, many surgeons prefer to kinematic alignment in TKA, as they wish to recreate the normal knee joint kinematics (19). However, 
Table 1 Demographics of the patients enrolled in the study

\begin{tabular}{lccc}
\hline Parameters & Navigation group & Conventional group & P value \\
\hline No. of patients & 39 & 40 & $65.33 \pm 7.59$ \\
Age (years) & $65.28 \pm 6.77$ & $31 / 9$ & 0.9789 \\
Sex (female/male) & $30 / 9$ & $21 / 19$ & 0.9513 \\
Affected extremity (left/right) & $15 / 24$ & 10.3 & 0.2104 \\
Allergic history (\%) & 17.9 & 92.5 & 0.3291 \\
Concomitant disease (\%) & 79.5 & 0.0949 \\
\hline
\end{tabular}

Table 2 Mechanical axis results of the two groups

\begin{tabular}{lccc}
\hline Parameters & Navigation group & Conventional group & $P$ value \\
\hline Outlier of neutral mechanical axis $\left(^{\circ}\right)$ & $1.60 \pm 1.11$ & $2.30 \pm 2.06$ & 0.0917 \\
Outlier $\leq \pm 3^{\circ}(\%)$ & 97.4 & 87.5 & 0.2007 \\
$\alpha$ angle $\left(^{\circ}\right)$ & $89.81 \pm 1.21$ & $89.76 \pm 2.20$ & 0.9922 \\
\hline
\end{tabular}

Table 3 Surgical related parameters

\begin{tabular}{|c|c|c|c|}
\hline Parameters & Navigation group & Conventional group & $P$ value \\
\hline Blood loss (mL) & $105.13 \pm 58.66$ & $113.00 \pm 71.55$ & 0.8825 \\
\hline Total adverse event (\%) & 59.0 & 67.5 & 0.4319 \\
\hline Anemia (\%) & 30.8 & 55.0 & 0.0297 \\
\hline Abnormal liver function (\%) & 10.3 & 15.0 & 0.7370 \\
\hline Severe adverse events (PE) (\%) & 0.0 & 2.5 & 1 \\
\hline
\end{tabular}

no consensus towards the functional outcomes between mechanically and kinematically aligned TKA (20-22). A level I prospective randomized control study showed significantly better results in patients with kinematical alignment (22). A randomized controlled clinical trial demonstrated no difference in 2-year functional outcomes using kinematic versus mechanical alignment in TKA (20). Maybe, more studies aiming to figure out the different functions should be conducted to help with the development of new knee navigation system. We did not compare clinical and function scores in the present study, as the follow up period was too short. Some studies showed that the greater precision of the navigation system did not conclusively reflect as better knee function $(23,24)$. Little clinical advantage may be observed in computer-assisted navigation over conventional devices at early stage (25). While, there was comparable functional scores and implant survival rates between TKA performed with and without navigation system in the midterm $(23,24)$. Combined with previous studies and the radiographic results of the present study, we could speculate the function outcomes in i-JOIN group was at least not worse than that in conventional group.

i-JOIN knee navigation system has some advantages over conventional intramedullary device and CAS. First, i-JOIN knee navigation system is simple and easier to be used. I-JOIN device does not need large computers and has only two components to perform navigation, which is simpler than CAS and even common handheld navigation system such as KneeAlign and iAssit. And, the pins needed in CAS which may weaken the anterior cortex of femur and 
increase additional bleeding were avoided in i-JOIN $(26,27)$. Second, i-JOIN knee navigation system can help reduce blood loss. The current study showed that the patients in navigation group experienced similar intraoperative blood loss compared with that in control group. However, the patients in navigation group exhibited much less anemia rate. Ueyama et al. (28) demonstrated that no statistically significant differences between an accelerometer-based portal navigation system group and conventional technique group in blood loss was observed. However, Ikawa et al. showed navigation system could significantly reduce blood loss (14). In the current study, only intraoperative blood loss was measured. The hidden blood loss in navigation group may be much less, as compared with that in conventional group. No violation of intramedullary femoral canal may result in less hidden blood loss and less anemia rate in i-JOIN group. Third, the patients in i-JOIN group experienced comparable adverse rate compared with that in conventional group. And, no subject was detected with PE in i-JOIN group.

i-JOIN device also has some shortcomings. First, the operative time of navigation group was significantly longer than that of conventional group. The increased time for navigation in the present study was about 14 mins and shorter than that required for computer-assisted TKA, generally 20 mins (29-31). After the initial learning curve, we are sure that the operation time will be reduced in i-JOIN related TKA procedures. Second, this device is actually a "partial" navigation system as the proximal tibia resection was performed using conventional instrument, instead of being navigated.

This study has several limitations. First, the follow up of the study was only one week. The functional scores and the implants survival rate were not evaluated in the study. Second, only coronal plane alignment was measured in this study. The sagittal plane alignment that is also very important to help evaluate the accuracy of the navigation system was not measured. Third, only conventional technique was used to compare the accuracy and knee function with i-JOIN in the current study. Other kinds of navigation devices such as KneeAlign and iAssit should also be compared with i-JOIN. As previously mentioned, we did not calculate hidden blood loss in the present study is another shortcoming of the study.

In conclusion, this study demonstrates that i-JOIN knee navigation system is at least as accurate as the intramedullary guide in performing resection of the distal femur. It could be a valuable option to intramedullary guide without the disadvantage of violation of the femoral canal.

\section{Acknowledgments}

Funding: This work was supported by Natural Nature Science Foundation of China (NSFC, 81802196 , 81572129), the Projects of International Cooperation and Exchanges of NSFC (81420108021), Key Program of NSFC (81730067), Excellent Young Scholars NSFC (81622033), Jiangsu Provincial Key Medical Center Foundation, Jiangsu Provincial Medical Outstanding Talent Foundation, Jiangsu Provincial Medical Youth Talent Foundation, Jiangsu Provincial Key Medical Talent Foundation, Jiangsu Provincial Key Research and Development Foundation (BE2016608), and Nature Science Foundation of Jiangsu Province (BK20180127).

\section{Footnote}

Conflicts of Interest: The authors have no conflict of interest to declare.

Ethical Statement: The study was approved by the authors' Institutional Review Boards (No.2017-119-02) and has been approved on Clinical Trial Registry (ID: ChiCTR1900023608). The authors are accountable for all aspects of the work in ensuring that questions related to the accuracy or integrity of any part of the work are appropriately investigated and resolved.

\section{References}

1. Srivastava A, Lee GY, Steklov N, et al. Effect of tibial component varus on wear in total knee arthroplasty. Knee 2012;19:560-3.

2. Jeffery RS, Morris RW, Denham RA. Coronal alignment after total knee replacement. J Bone Joint Surg Br 1991;73:709-14.

3. Lording T, Lustig S, Neyret P. Coronal alignment after total knee arthroplasty. EFORT Open Rev 2017;1:12-7.

4. Brys DA, Lombardi AV, Mallory TH, et al. A comparison of intramedullary and extramedullary alignment systems for tibial component placement in total knee arthroplasty. Clin Orthop Relat Res 1991:175-9.

5. Ritter MA, Davis KE, Meding JB, et al. The effect of alignment and BMI on failure of total knee replacement. J Bone Joint Surg Am 2011;93:1588-96.

6. Anderson KC, Buehler KC, Markel DC. Computer 
Assisted Navigation in Total Knee Arthroplasty: Comparison With Conventional Methods. J Arthroplasty 2005;20:132-8.

7. Blakeney WG, Khan RJ, Wall SJ. Computer-assisted techniques versus conventional guides for component alignment in total knee arthroplasty: a randomized controlled trial. J Bone Joint Surg Am 2011;93:1377-84.

8. Matassi F, Cozzi Lepri A, Innocenti M, et al. Total Knee Arthroplasty in Patients With Extra-Articular Deformity: Restoration of Mechanical Alignment Using Accelerometer-Based Navigation System. J Arthroplasty 2019;34:676-81.

9. Nam D, Weeks KD, Reinhardt KR, et al. AccelerometerBased, Portable Navigation vs Imageless, Large-Console Computer-Assisted Navigation in Total Knee Arthroplasty: A Comparison of Radiographic Results. J Arthroplasty 2013;28:255-61.

10. Nam D, Nawabi DH, Cross MB, et al. Accelerometerbased computer navigation for performing the distal femoral resection in total knee arthroplasty. J Arthroplasty 2012;27:1717-22.

11. Kinney MC, Cidambi KR, Severns DL, et al. Comparison of the iAssist Handheld Guidance System to Conventional Instruments for Mechanical Axis Restoration in Total Knee Arthroplasty. J Arthroplasty 2018;33:61-6.

12. Goh GSH, Liow MHL, Lim WSR, et al. AccelerometerBased Navigation Is as Accurate as Optical Computer Navigation in Restoring the Joint Line and Mechanical Axis After Total Knee Arthroplasty: A Prospective Matched Study. J Arthroplasty 2016;31:92-7.

13. Huang EH, Copp SN, Bugbee WD. Accuracy of A Handheld Accelerometer-Based Navigation System for Femoral and Tibial Resection in Total Knee Arthroplasty. J Arthroplasty 2015;30:1906-10.

14. Ikawa T, Takemura S, Kim M, et al. Usefulness of an accelerometer-based portable navigation system in total knee arthroplasty. Bone Joint J 2017;99-b:1047-52.

15. Maderbacher G, Schaumburger J, Keshmiri A, et al. Pinless navigation in total knee arthroplasty: Navigation reduced by the maximum? Int Orthop 2015;39:455-60.

16. Chen JY, Chin PL, Li Z, et al. Radiological outcomes of pinless navigation in total knee arthroplasty: a randomized controlled trial. Knee Surg Sports Traumatol Arthrosc 2015;23:3556-62.

17. Liow MH, Goh GS, Pang HN, et al. Computer-assisted stereotaxic navigation improves the accuracy of mechanical alignment and component positioning in total knee arthroplasty. Arch Orthop Trauma Surg 2016;136:1173-80.
18. Shah NA, Patil HG, Dhawale AS, et al. Limited Femoral Navigation Versus Conventional Intramedullary Femoral Jig Based Instrumentation for Achieving Optimal Restoration of Mechanical Axis Post Total Knee Arthroplasty: A Prospective Comparative Study of 200 Knees. J Arthroplasty 2015;30:559-63.

19. Karuppal R. Kinematic alignment in total knee arthroplasty: Does it really matter? J Orthop 2016;13:A1-A3.

20. Young SW, Walker ML, Bayan A, et al. The Chitranjan S. Ranawat Award : No Difference in 2-year Functional Outcomes Using Kinematic versus Mechanical Alignment in TKA: A Randomized Controlled Clinical Trial. Clin Orthop Relat Res 2017;475:9-20.

21. Yoon JR, Han SB, Jee MK, et al. Comparison of kinematic and mechanical alignment techniques in primary total knee arthroplasty: A meta-analysis. Medicine (Baltimore) 2017;96:e8157-e.

22. Dossett HG, Swartz GJ, Estrada NA, et al. Kinematically versus mechanically aligned total knee arthroplasty. Orthopedics 2012;35:e160-9.

23. Hernández-Vaquero D, Suarez-Vazquez A, IglesiasFernandez S. Can computer assistance improve the clinical and functional scores in total knee arthroplasty? Clin Orthop Relat Res 2011;469:3436-42.

24. Harvie P, Sloan K, Beaver RJ. Computer navigation vs conventional total knee arthroplasty: five-year functional results of a prospective randomized trial. J Arthroplasty 2012;27:667-72.e1.

25. Cheng T, Pan X-Y, Mao X, et al. Little clinical advantage of computer-assisted navigation over conventional instrumentation in primary total knee arthroplasty at early follow-up. Knee 2012;19:237-45.

26. Kamara E, Berliner ZP, Hepinstall MS, et al. Pin Site Complications Associated With Computer-Assisted Navigation in Hip and Knee Arthroplasty. J Arthroplasty 2017;32:2842-6.

27. Minoda $\mathrm{Y}$, Watanabe $\mathrm{K}$, Iwaki $\mathrm{H}$, et al. Theoretical risk of anterior femoral cortex notching in total knee arthroplasty using a navigation system. J Arthroplasty 2013;28:1533-7.

28. Ueyama H, Minoda Y, Sugama R, et al. An accelerometerbased portable navigation system improved prosthetic alignment after total knee arthroplasty in 3D measurements. Knee Surg Sports Traumatol Arthrosc 2019;27:1580-6.

29. Decking R, Markmann Y, Fuchs J, et al. Leg Axis After Computer-Navigated Total Knee Arthroplasty: A Prospective Randomized Trial Comparing ComputerNavigated and Manual Implantation. J Arthroplasty 
2005;20:282-8.

30. Bäthis H, Perlick L, Tingart M, et al. Alignment in total knee arthroplasty. A comparison of computer-assisted surgery with the conventional technique. J Bone Joint Surg
Br 2004;86:682-7.

31. Martin A, Wohlgenannt O, Prenn M, et al. Imageless navigation for TKA increases implantation accuracy. Clin Orthop Relat Res 2007;460:178-84.

Cite this article as: $\mathrm{Xu} X$, Liu P, Yuan Z, Wang D, Lu Q, Zhang Z, Jiang Q, Shi D. Comparison of a novel handheld accelerometer-based navigation system and conventional instrument for performing distal femoral resection in total knee arthroplasty: a randomized controlled trial. Ann Transl Med 2019;7(22):659. doi: 10.21037/atm.2019.10.55 